\title{
LES OLIGOCHẼTES AQUATIQUES DU SUD-OUEST DE LA FRANCE
}

\author{
par N. Giani ${ }^{1}$.
}

A partir de prélèvements effectués dans une centaine de stations, l'auteur dresse une liste de 47 espèces d'Oligochètes vivant dans les milieux aquatiques du sud-ouest de la France. Ces espèces appartiennent à 6 familles parmi lesquelles celles des Naididae (27 sp.) et des Tubificidae sont les mieux représentées. L'auteur envisage ensuite la répartition des espèces dans les Pyrénées.

\section{The aquatic oligochaetes from the southwest fo France.}

From samples taken at about a hundred stations, the author draws up a list of 47 species of oligochaetes living in the aquatic environments of the southwest of France. These species are distributed in six families with the greatest number of species in the Naididae $(27$ sp.) and Tubificidae. The author examines the distribution of the species in the Pyrenees.

Les Oligochètes aquatiques ont été fort peu étudiés en France jusqu'à ces dernières années. Cependant les Oligochètes dulçaquicoles de l'Est de la France sont bien connus grâce aux travaux de Tétry (1938) en Lorraine et surtout de Juget (1957 et 1959) dans la région Saône-Rhône. Ce dernier a particulièrement étudié le peuplement du lac Léman (Juget, 1958 et 1967), du lac d'Annecy (Juget, 1958) et des étangs de la Dombes (Amoros et Juget ,1970).

En ce qui concerne le Sud et l'Ouest de la France, Lasserre (1966 et 1967) a dressé un inventaire des Oligochètes marins des côtes de ces régions. Juget et Giani (1974) ont établi une première liste des Oligochètes peuplant les milicux aquatiques de la chaîne pyrénéenne. Le présent travail a pour objet de compléter ce relevé faunistique et de l'étendre à tout le sud-ouest de la France.

Le matérieli2 examiné ici provient de mares, lacs, ruisseaux ou rivières situés dans douze départements du sud-ouest de la France : Ariège, Aude, Aveyron, Cantal, Haute-Garonne, Landes, Lot, Lotet-Garonne, Lozère, Pyrénées-Atlantiques, Hautes-Pyrénées, Tarn. La localisation des diverses stations est précisée par les figures 1 et 2. La plupart de ces stations ont déjà été décrites par de nom-

1. Laboratoire d'Hydrobiologie, Université Paul-Sabatier, 118, route de Narbonne, 31077 Toulouse Cedex, France.

2. Je remercie M. A. Thomas qui m'a légué une partie du matériel examiné ici et m'a fait bénéficier de sa connaissance des cours d'eau du sud-ouest de la France.

Je remercie également MM. Cassan, Laur, Pujol du laboratoire de Toulouse é M. Dumas du laboratoire de l'I.N.R.A. à Biarritz qui m’ont confié pour détermination nombre des Oligochètes cités ici. 


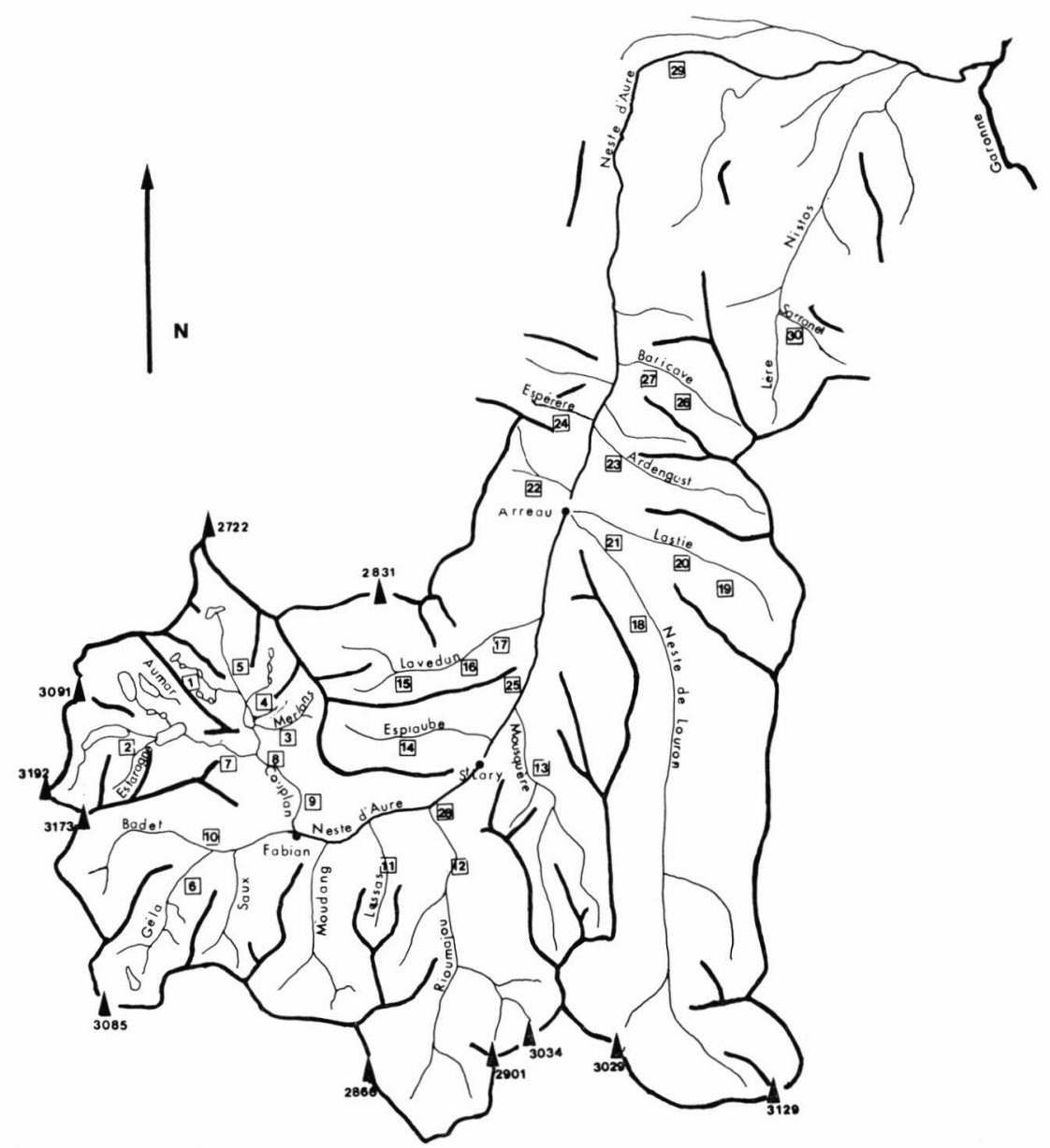

Fig. 1. - Répartition des stations de prélèvements dans la vallée d'Aure.

breux auteurs et notamment Berthélemy (1966), Décamps (1967), Lavandier (1974), Pujol (1975), Berthélemy et Laur (1975), Thomas (1976) et nous renverrons à ces travaux pour plus ample information.

\section{1. - LISTE DES STATIONS}

— Vallée d’Aure (fig. 1).

1: Tourbière d'Aumar (alt. $2300 \mathrm{~m}$ ); 2 : Ruisseau déversoir lac de l'Ile $(2240 \mathrm{~m})$; 3 : Ruisseau de Merlans $(2000 \mathrm{~m}) ; 4$ : R. des Pins (1900 m); 5 : R. de Port-Bielh $(1850 \mathrm{~m}) ; 6$ : Neste de la Géla $(1450 \mathrm{~m})$; 7 : N. de 
Couplan à Artigusse $(1410 \mathrm{~m}), 8$ : à $1380 \mathrm{~m}, 9:$ à $1210 \mathrm{~m}$; 10 : N. de Badet $(1500 \mathrm{~m})$; 11 : R. de Lassas $(900 \mathrm{~m})$; 12 : R. de Rioumajou (1 $100 \mathrm{~m})$; 13 : R. de la Mousquère $(1400 \mathrm{~m}) ; 14: \mathrm{R}$. d'Espiaube $(1600 \mathrm{~m}) ; 15: \mathrm{R}$. de Lavedan $(1800 \mathrm{~m}), 16: 1350 \mathrm{~m}, 17$ : à $900 \mathrm{~m} ; 18: \mathrm{N}$. de Louron (960 m); 19 : R. de Lastie $(1600 \mathrm{~m}), 20$ : à $1060 \mathrm{~m}, 21$ : à $750 \mathrm{~m}$; 22 : R. d'Aspin $(800 \mathrm{~m})$; 23 : R. d'Ardengost $(660 \mathrm{~m})$; 24 : R. de l'Espérère $(670 \mathrm{~m})$; 25 : Cressonnière d'Agos $(770 \mathrm{~m}) ; 26: \mathrm{R}$. de Baricave $(1000 \mathrm{~m}), 27$ : à $700 \mathrm{~m}$; 28 : N. d'Aure à Tramezaygues $(900 \mathrm{~m}), 29:$ au Pont de Bisous (490 m); 30 : R. de Nistos $(470 \mathrm{~m})$.

\section{- Autres stations (fig. 2).}

31 : La Garonne à Portet $(150 \mathrm{~m}), 32$ : à Carbonne $(202 \mathrm{~m}), 33$ : au barrage de Boussens $(270 \mathrm{~m}), 34$ : en amont du Salat $(290 \mathrm{~m}), 35$ : à Chaum $(471 \mathrm{~m})$; 36 : la Pique (affluent de la Garonne) à 600, 37 : à $530 \mathrm{~m}, 38$ : au barrage de Cazaux $(500 \mathrm{~m})$; 39 : Fontaine de Guindoulé (Bassin du Salat) (1 $260 \mathrm{~m})$; 40 : l'Arac (affl. du Salat) à $(470 \mathrm{~m}), 41$ : à $650 \mathrm{~m} ; 42$ : Fontaine de la Piche (B. du Salat) $(970 \mathrm{~m}) ; 43:$ R. d'Erp (affl. du Salat) $(720 \mathrm{~m}) ; 44:$ R. des

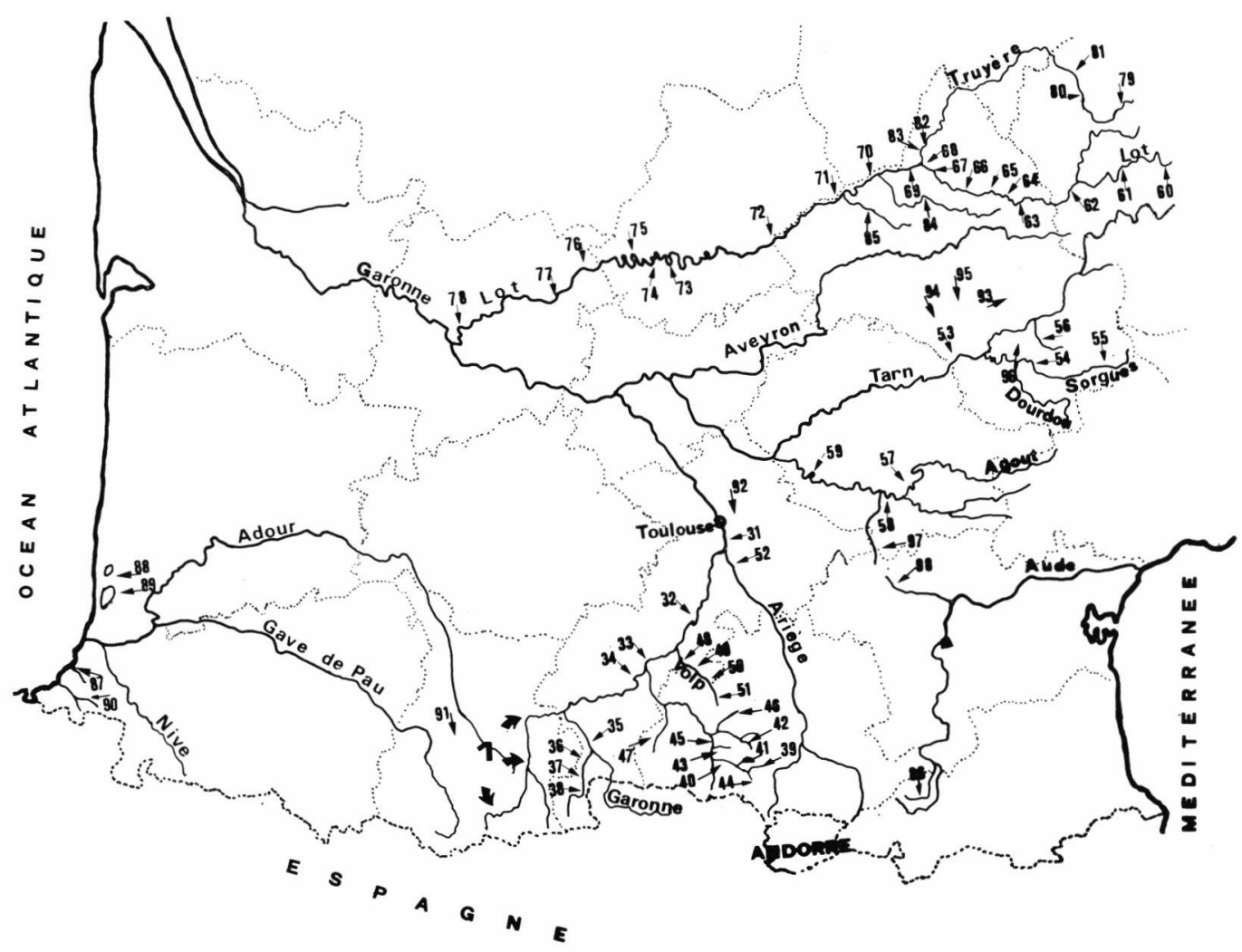

FIG. 2. - Répartition des stations de prélèvements dans le Sud-Ouest de la France. La zone 1 correspond à la figure 1 . 
Cors (affl. du Salat) $(830 \mathrm{~m}) ; 45$ : le Salat (affl. de la Garonne) (415 m); 46: R. de Perri (B. du Salat) $(413 \mathrm{~m}) ; 47:$ R. de l'Escalette (B. du Salat) $(410 \mathrm{~m}) ; 48$ : le Volp (affl. de la Garonne) à $240 \mathrm{~m}, 49$ : à $270 \mathrm{~m}, 50$ : à $290 \mathrm{~m}, 51$ : à $460 \mathrm{~m}$; 52 : l'Ariège (affl. de la Garonne) à $160 \mathrm{~m}$; 53 : le Tarn (affl. de la Garonne) à Broquiès $(270 \mathrm{~m}) ; 54$ : la Sorgues (affl. du Tarn) à $340 \mathrm{~m}, 55$ : à $580 \mathrm{~m}$ (Résurgence); 56 : le Cernon (affl. du Tarn) $(417 \mathrm{~m})$; 57 : l'Agoût (affl. du Tarn) à $172 \mathrm{~m}, 58:$ à $165 \mathrm{~m}, 59$ : à $140 \mathrm{~m}$; 60 : le Lot (affl. de la Garonne) près de la source $(1085 \mathrm{~m}), 61$ : à $710 \mathrm{~m}, 62$ : à $524 \mathrm{~m}, 63$ : à $425 \mathrm{~m}, 64$ : barrage de Castelnau $(410 \mathrm{~m}), 65$ : à $346 \mathrm{~m}$, 66 : à $312 \mathrm{~m}, 67$ : barrage de Golhinac $(300 \mathrm{~m}), 68:$ à $221 \mathrm{~m}, 69:$ à $214 \mathrm{~m}$, 70 : aval Dourdou $(187 \mathrm{~m}), 71$ : amont du Riou-Mort $(176 \mathrm{~m}), 72$ : barrage de Carjac $(145 \mathrm{~m}), 73$ : à $104 \mathrm{~m}, 74$ : barrage de Luzech $(102 \mathrm{~m}), 75$ : à $78 \mathrm{~m}$, 76 : à $65 \mathrm{~m}, 77$ : barrage de Villeneuve $(40 \mathrm{~m}), 78$ : barrage du Temple $(38 \mathrm{~m})$; 79 : la Truyère (affl. du Lot) à $(1224 \mathrm{~m}), 80:$ à $929 \mathrm{~m}, 81$ : à $865 \mathrm{~m}$, 82 : barrage de Cambeyrac $(230 \mathrm{~m}), 83$ : à $221 \mathrm{~m} ; 84$ : le Dourdou (affl. du Lot) $(200 \mathrm{~m})$; 85 : le Riou-Mort (affl. du Lot) (261 m) ; 86 : la Lladure (affl. de l'Aude) $(1500 \mathrm{~m}) ; 87:$ R. de Guétary $(10 \mathrm{~m}) ; 88$ : Etang Blanc $(40 \mathrm{~m})$; 89 : Etang d'Hossegor $(03 \mathrm{~m}) ; 90$ : Bassins d'élevage de Saint-Pé-sur-Nivelle $(90 \mathrm{~m})$; 91 : source près de Bagnères-de-Bigorre $(780 \mathrm{~m})$; 92 : mare près de Toulouse $(200 \mathrm{~m}) ; 93$ : fontaine près de Villefranche-de-Panat $(800 \mathrm{~m}) ; 94$ : Etang de Durenque $(725 \mathrm{~m})$; 95 : Mare près de Réquista $(662 \mathrm{~m})$; 96 : Mare près de Saint-Affrique $(305 \mathrm{~m})$; 97 : Déversoir du lac des Cammazes (affl. de l'Agoût) $(560 \mathrm{~m}) ; 98: \mathrm{R}$. de Villespy (affl. de l'Aude) $(180 \mathrm{~m})$.

\section{2. - LISTE FAUNISTIQUE}

Pour chaque espèce nous préciserons :

- le nombre $n$ d'individus examinés,

- les stations où l'espèce a été récoltée,

- la répartition mondiale de l'espèce selon Brinkhurst et al. (1971) pour les Lumbriculidae, les Naididae et les Tubificidae,

- les diverses observations de l'espèce en France.

\section{1. - ORDRE LUMBRICULIDA.}

\section{FAMILLE DES LUMBRICULIDAE :}

Lumbriculus variegatus (Müller, 1774) $\mathrm{n}=\mathbf{5 8 0}$.

Répartition : hémisphère nord, Afrique du Sud, Nouvelle-Zélande.

France : Pyrénées (Despax, 1927 ; Juget et Giani, 1974) ; Garonne (Despax, 1916) ; Lac Léman (Juget, 1958 et 1967) ; Dombes (Amoros et Juget, 1970) ; Canal de Roubaix (Schodduyn, 1925) ; Flandre (Schodduyn, 1926).

Stations : $1,3,32,34,36,38,52,57,58,61,62,64,66,69,70$, $72,73,74,76,80,83,84,88,90,94,95$.

Espèce rencontrée jusqu'à $2300 \mathrm{~m}$ où elle peut être très abondante dans les lacs et les tourbières. Elle est fréquente dans les milieux riches en matières organiques : tourbières, lacs, étangs, 
mares, zones d'eaux calmes dans les rivières. Elle est absente dans les torrents et la partie supérieure des cours d'eau c'est-à-dire dans la zone où le courant empêche le dépôt de vase. Nous n'avons jamais rencontré cette espèce en phase de reproduction sexuée.

Stylodrilus heringianus Claparède, $1862 \mathrm{n}=209$.

Répartition : Europe, Asie occidentale, Amérique du Nord.

France : Lorraine (Tétry, 1938) ; Léman (Juget, 1958 et 1967) ; Lac d'Annecy (Juget, 1958) ; Pyrénées (Juget et Giani, 1974); Alsace (Schweitzer, 1935).

Stations : 1, 2, 14, 15, 20,21, 23, 24, 29, 30, 32, 39, 40, 41, 44, $45,47,48,49,50,51,52,60,61,63,65,66,79,80,81,82,97$.

Nous avons récolté cette espèce jusqu'à $2000 \mathrm{~m}$ d'altitude dans les torrents et jusqu'à $2300 \mathrm{~m}$ dans les tourbières et les lacs. Elle est abondante dans les torrents pyrénéens où elle vit sous les pierres en compagnie d'Eiseniella tetraedra notamment. En plaine elle colonise de la même façon la partie supérieure des cours d'eau, là où la pente est assez forte pour empêcher l'envasement. Nous avons observé des individus matures depuis le mois de mars jusqu'au mois de septembre.

\section{2. - ORDRE HAPLOTAXIDA.}

\subsection{1. - FAMILLE DES HAPLOTAXIDAE}

Haplotaxis gordioides (Hartmann, 1821) $n=45$.

Répartition : holarctique.

France : Bretagne (Ferronnière, 1889) ; Nord (Moniez, 1888; Giard, 1882) ; Lorraine (Brunotte, 1892 ; Rémy, 1932 ; Tétry, 1938) ; Alsace (Hartmann, 1923 ; Moszinsky, 1938) ; Vosges (Brunotte, 1892 ; Rémy, 1932) ; Haute Tarantaise (Hubault, 1927) ; Ille-et-Vilaine (Pesson, 1935) ; Creuse (Giard, 1889) ; Corse (Rémy, 1943 ) ; Pyrénées-Orientales (Vandel, 1922); Lac Léman (Juget, 1958 el 1967 ).

Stations : 8, 9, 11, 12, 13, 17, 19, 21, 26, 28, 42, 79, 86, 98.

Nous avons noté la présence de cette espèce entre 700 et $1600 \mathrm{~m}$ d'altitude dans les torrents pyrénéens où elle est constante mais jamais très abondante ( 15 individus maximum par mètre carré). En plaine nous l'avons rencontrée près des sources des cours d'eau.

Généralement signalée dans les puits, le fond des lacs, et surtout la terre humide cette espèce est souvent considérée comme accidentelle dans les milieux aquatiques. Sa présence constante dans les torrents de montagne laisse penser que cette forme est certainement sténotherme d'eau froide. 


\subsection{2. - FAMILLE DES NAIDIDAE}

\subsubsection{1. - Sous-famille des Chaetogastrinae : SPERBER, 1948.}

Chaetogaster diastrophus (Gruithuisen, 1828) $\mathrm{n}=\mathbf{1 6}$.

Répartition : Europe, Inde, Chine, Afghanistan, Amérique du Nord et du Sud.

France : Lorraine (Tétry, 1938), Lac Léman (Juget, 1958 et 1967 ) ; Dombes (Amoros et Juget, 1970) ; Pyrénées (Juget et Giani, 1974) ; Flandre (Schodduyn, 1926).

Stations : 49, 52, 56.

Chaetogaster diaphanus (Gruithuisen, 1828) $\mathrm{n}=10$.

Répartition : Europe, Asie, Amérique du Nord.

France : Lorraine (Tétry, 1938) ; Lac Léman (Juget, 1967) ; le Rhône (Lafon, 1953) ; Dombes (Amoros et Juget, 1970) ; Pyrénées (Juget et Giani, 1974) ; Marne (Cohen, 1941) ; Canal de Roubaix (Schodduyn, 1925).

Stations 92.

Chaetogaster langi Brelscher, $1896 \mathrm{n}=18$.

Répartition: Europe, Asie, Afrique, Amérique.

France : le Rhône (Lafon, 1953) ; Lac Léman (Jugel, 1967) ; Pyrénées (Juget et Giani, 1974).

Stations : $49,92$.

Chaetogaster setosus Sretlov, $1925 \mathrm{n}=8$.

Répartition : Russie.

France : Léman (Ch. setosus var. lemani, Juget 1967).

Stations : 8 individus le $26-2-76$ dans le Volp (49).

Décrite de la rivière Khama en Russie cette espèce a été retrouvée dans les zones sub-littorale et profonde du lac Léman (Juget, 1967). Les individus récoltés dans le Volp sont très proches de Ch. setosus var. lemani (Juget, 1967) ; ils possèdent 7 soies par faisceaux sur le segment II et 4 soies au-delà du segment VI, alors que la forme type possède 9-10 soies en II et 5-8 soies audelà de VI. Juget (1967) considère celte espèce comme une relicte glaciaire chez laquelle la fragmentation de l'aire de répartition due au retrait des glaciers aurait conduit par isolement géographique à la différenciation intraspécifique.

Chaetogaster limnaei Von Baer, $1827 \mathrm{n}=6$.

Répartition : Europe, Asie, Amérique du Nord.

France : Lorraine (Tétry, 1938) ; l'Yonne et la Marne (Cohen, 1941 ) ; le Rhône (Lafon, 1953) ; lac Léman (Jugct, 1967) ; Flandre (Schodduyn, 1924).

Stations : 32. 
Nous avons récolté cette espèce dans la cavité pulmonaire de limnées provenant de la Garonne. Compte tenu de la biologie très particulière de cette forme nous ne l'avons que très peu recherchée, aussi doit-elle être plus répandue que nos récoltes le laissent envisager.

\subsubsection{2. - Sous-famille Paranaidinae Sperber, 1948.}

Paranais litoralis (Müller, 1784) $\mathrm{n}=18$.

Répartition : Afrique, Europe, Asie, Amérique du Nord.

France : Arcachon (Amanieu, 1969 ; Lasserre, 1966) ; Roscoff, Penpoull, Concarneau (Lasserre, 1967) ; La Houque (Manche) (Delphy, 1921).

Récoltes : Espèce des eaux saumâtres et salées dont nous avons récolté 21 individus le 25-1-76 dans l'étang salé d'Hossegor (89).

\subsubsection{3. - Sous-Famille Naidinae Lastockin, 1924.}

Specaria josinae (Vejdovsky, 1883) $n=150$.

Répartition : Europe, Asie, Amérique du Nord.

France : Lac Léman (Juget, 1967) ; Pyrénées (Juget et Giani, 1974).

Stations : $67,72,75,82,86,90$.

A l'exception de la station 86 cetle espèce a été récoltée essentiellement dans les sédiments des lacs de barrage.

Uncinais uncinata (Orsted, 1842) $n=40$.

Répartition : Amérique du Nord, Europe, Turkestan.

France : Lac Léman (Juget, 1958 ct 1967) ; lac d'Annecy (Juget, 1958 ) ; Pyrénées (Juget et Giani, 1974) ; Yonne (Cohen, 1941).

Stations : $69,82,90$.

Cette forme vit généralement dans la vase de la zone littorale des barrages (82) ou des eaux calmes $(69,90)$.

Ophidonais serpentina (Müller, 1773) $\mathrm{n}=284$.

Répartition : Europe, Amérique du Nord et du Sud, Sibérie, Turquie.

France : Lorraine (Tétry, 1938) ; Lac Léman (Juget, 1967) ; Saône (Juget, 1957) ; Dombes (Amoros et Juget, 1970) ; Flandres (Schodduyn, 1926).

Stations : $31,56,63,65,88$.

Cette espèce non encore trouvée dans les Pyrénées est fréquente dans la végétation immergée.

Nais communis Piguet, $1906 \mathrm{n}=196$.

Répartition : cosmopolite.

France : Lorraine (Tétry, 1938) ; la Marne (Cohen, 1941) ; lac 
Léman (Juget, 1967) ; Dombes (Amoros et Juget, 1970) ; Pyrénées (Juget et Giani, 1974).

Stations : $1,7,37,38,39,43,49,56,81,82,86,96$.

Notée jusqu'à $2300 \mathrm{~m}$ d'altitude cette espèce est très ubiquiste I uisqu'elle vit aussi bien dans les tourbières, les eaux stagnantes ou courantes. Elle est abondante dans les torrents pyrénéens jusqu'à $1600 \mathrm{~m}$ d'altitude où elle vit en compagnie de Nais alpina.

Nais variabilis Piguet, $1906 n=8$.

Répartition : cosmopolite.

France : Lorraine (Tétry, 1938) ; Pyrénées (Monard, 1928) ; Meudon (Cohen, 1941) ; lac Léman (Juget, 1967) ; Dombes (Amoros et Juget, 1970).

Stations : 47 le 14-4-1975.

C'est la seule espèce d'Oligochète mentionnée dans la Limnofauna Europaea (Brinkhurst, 1966) pour la zone pyrénéenne. Cette espèce semble pourtant assez rare dans le Sud-Ouest de la France ; elle n'a pas été rencontrée par Juget et Giani (1974) dans le Massif du Néouvielle.

Nais simplex Piguet, $1906 \mathrm{n}=4$.

Répartition : Europe, Amérique du Nord, Afrique, Tibet ?.

France : Lorraine (Tétry, 1938) ; lac Léman (Juget, 1967) ; Dombes (Amoros et Juget, 1970) ; Pyrénées (Juget et Giani, 1974) . Stations : 92 le 14-1-76.

Nais bretscheri Michaelsen, $1899 \mathrm{n}=63$.

Répartition : Europe, Turkestan.

France : la Marne (Cohen, 1941) ; lac Léman (Juget, 1967) ; Flandre (Schodduyn, 1926).

Stations : $31,46,49,52,62,64,86,90,92$.

Récoltée jusqu'à $1600 \mathrm{~m}$ d'altitude cette espèce est nouvelle pour les Pyrénées.

Nais pardalis Piguet, $1906 \mathrm{n}=27$.

Répartition : Europe, Asie, Amérique du Nord et du Sud.

France : Lorraine (Tétry, 1938) ; le Rhône (Lafon, 1953) ; lac Léman (Juget, 1967).

Stations : $31,48,49,50,64,90,92,96$.

Nous n'avons pas rencontré cette espèce au-dessus de $700 \mathrm{~m}$ d'altitude.

Nais elinguis Müller, $1773 \mathrm{n}=326$.

Répartition : cosmopolite.

France : Nord (Moniez, 1888) ; Flandre (Schodduyn, 1926) ; Lorraine (Florentin, 1899 ; Tétry, 1938) ; bassin d'Arcachon (Lasserre, 1966) ; lac Léman (Juget, 1967) ; lac d'Annecy (Le Roux, 1907 ) ; lac de iLourdes (Schodduyn, 1926). 
Stations : $1,29,31,47,51,52,56,46,61,62,63,65,66,86,87$. Nais elinguis est certainement le Naididae le plus abondant dans le Sud-Ouest de la France. C'est une forme très ubiquiste qui vit également en eaux saumâtres. Elle est nouvelle pour les Pyrénées où nous l'avons observée jusqu'à $2300 \mathrm{~m}$ d'altitude dans une tourbière et jusqu'à $1600 \mathrm{~m}$ en eau courante.

Nais alpina Sperber, $1948 \mathrm{n}=41$.

Répartition : Europe.

Stations : 2, 5, 29, 37, 42, 43, 44, 46, 49, 60, 81, 86.

Forme boréo-alpine d'eau courante, nouvelle pour la France et les Pyrénées. C'est une espèce constante dans les torrents pyrénéens où elle est généralement le seul Naididae au-dessus de $1600 \mathrm{~m}$; nous l'avons rencontrée jusqu'à $2000 \mathrm{~m}$. En dessous de $1600 \mathrm{~m}$, elle coexiste fréquemment avec $N$. communis. C'est une forme sténotherme d'eau froide que l'on rencontre en plaine dans la partie supérieure des cours d'eau, au voisinage de la source.

Nais barbata Müller, $1773 \mathrm{n}=36$.

Répartition :Europe, Asie, Amérique du Nord, Afghanistan.

France :lac Léman (Juget, 1967).

Stations : $48,49,82,90$.

Nais pseudobtusa Piguet, $1906 \mathrm{n}=11$.

Répartition : Europe, Amérique du Nord, Afrique, Afghanistan.

France : Vendée (Ferronnière, 1899); Vosges (Moszinsky, 1938); lac Léman (Juget, 1967).

Stations : 86.

Espèce nouvelle pour les Pyrénées où nous l'avons prélevée à $1600 \mathrm{~m}$ d'altitude.

Slavina appendiculata d'Udekem, $1855 \mathrm{n}=32$.

Répartition : Europe, Amérique du Nord et du Sud, Asie orientale et du Sud, Afrique, Nouvelle-Zélande.

France : lac Léman (Juget, 1967) ; Dombes (Amoros et Juget, 1970).

Stations : $25,82,86$.

Espèce nouvelle pour la faune des Pyrénées; récoltée jusqu'à $1600 \mathrm{~m}$ d'altitude.

Vejdovskyella comata (Vejdovsky, 1883) $\mathrm{n}=28$.

Répartition : Europe, Asie, Amérique du Nord, Afrique.

France : environs de Paris (Richard, 1897) ; Dombes (Amoros et Juget, 1970) ; Pyrénées (Juget et Giani, 1974) ; lac de Lourdes (Schodduyn, 1926).

Stations : $82,86,90$.

Ripistes parasita (Schmidt, 1847) $\mathrm{n}=\mathbf{2}$.

Répartition : Europe, lac Baïkal. 
France : Bretagne (Pesson, 1938) ; Dombes (Amoros et Juget, $1970)$.

Récoltes : 2 individus prélevés le 25-1-76 dans l'Étang-Blanc (88).

Stylaria lacustris (Linné, 1767) $\mathrm{n}=458$.

Répartition : Europe, Asie occidentale, Amérique du Nord.

France : Flandre (Schodduyn, 1926) ; Lorraine (Tétry, 1938) ; lac Léman (Juget, 1967) ; Dombes (Amoros el Juget, 1970). Camargue (Aguesse, 1957) ; Yonne (Cohen, 1941) ; Canal de Roubaix (Schodduyn, 1925) ; lac d'Annecy (Le Roux, 1907).

Stations : $63,65,82,83,88,94,96$.

Espèce de plaine qui vit essentiellement en eau stagnante; jamais récoltée dans les Pyrénées.

Dero digitata (Müller, 1773) $\mathrm{n}=106$.

Répartition : cosmopolite.

France : lac Léman (Juget, 1967) ; Dombes (Amoros et Juget, 1970 ) ; Lorraine (Tétry, 1938).

Stations : $63,64,67,72,82,92$.

Forme essentiellement d'eau stagnante que nous n'avons pas rencontrée dans les Pyrénées.

Aulophorus furcatus (Müller, 1773) $\mathrm{n}=9$.

Répartition :cosmopolite.

France : ?.

Stations : 92.

\subsubsection{4. - Sous-famille Pristininae Iastockin, 1924.}

Pristina bilobata (Bretscher, 1908) $\mathrm{n}=1$.

Répartition : Europe, Palestine, Turkestan, Afrique.

Récoltes : 1 individu le 15-1-76 dans un prélèvement de l'Étang Blanc (88) dans les Landes.

France : Yonne (Cohen, 1941) ?.

Pristina foreli (Piguet, 1906) $\mathrm{n}=3$.

Répartition : Europe, Turkestan, Japon ?, Afrique.

France : lac Léman (Juget, 1967).

Stations : 50, 90, 92.

Pristina longiseta Ehrenberg, $1828 \mathrm{n}=1$.

Répartition : Europe, Asic, Afrique, Australie.

France : lac Léman (Juget, 1967) ; Dombes (Amoros et Juget, 1970 ) ; lac de Lourdes (Schodduyn, 1926).

Stations : 90. 


\subsection{3. - FAMille Des TUBIFICIDAE}

2.2.3.1. - Sous-famille Tubificinae Eisen, 1879.

Tubifex tubifex (Müller, 1774) $\mathrm{n}=303$.

Répartition cosmopolite.

France : Flandre (Schodduyn, 1926) ; Lorraine (Tétry, 1938) ; S€ine, Yonne, Marne (Cohen, 1941) ; Rhône (Lafon, 1953) ; Saône (Juget, 1957) ; lac Léman (Juget, 1958 et 1967) ; lac d'Annecy (Le Roux, 1907 ; Juget, 1958) ; Dombes (Amoros et Juget, 1970) ; Pyrénées (Juget et Giani, 1974).

Stations : $54,55,56,61,62,63,69,70,71,72,82,83,84,86$, $94,95,91,90,96,98$.

Cette espèce est fréquente dans tous les milieux d'eau stagnante ; en eau courante elle se rencontre dans les zones où la pente et la vitesse du courant permettent une accumulation de vase fine. Ainsi, cette forme est pratiquement absente dans les torrents pyrénéens alors que Juget et Giani (1974) l'ont prélevée en abondance dans les lacs de haute altitude.

Tubifex ignotus (Stolc, 1886) $\mathrm{n}=\mathbf{3 6}$.

Répartition : Europe, Amérique du Nord, Afrique du Sud.

France : Pyrénées (Juget et Giani, 1974).

Stations : 31, 38, 60, 61, 62, 63, 69, 70, 71, 72, 73, 79, 82, 83, 85, $90,94$.

T. ignotus est un Tubificidae caractéristique des eaux courantes bien qu'il ne forme jamais des populations très denses. Nous l'avons fréquemment rencontré dans les sédiments sablonneux des parties supérieures des cours d'eau en compagnie de Limnodrilus hoffmeisteri et surtout de Stylodrilus heringianus.

Limnodrilus hoffmeisteri Claparède, $1862 \mathrm{n}=244$.

Répartition : cosmopolite.

France : Saône (Juget, 1957) ; lac Léman (Juget, 1958 et 1967) ; Dombes (Amoros et Juget, 1970) ; Lorraine (Tétry, 1938).

Stations : 5, 48, 52, 59, 63, 66, 67, 68, 70, 71, 72, 73, 76, 77, 78, $80,81,82,83,84,86,87,88,90,91,94,96$.

Cette forme est la plus abondante des espèces du genre Limnodrilus dans le Sud-Ouest de la France. En eaux stagnantes elle vit fréquemment dans la vase avec $T$. tubifex, alors qu'en eau courante elle est plus fréquente dans les sédiments sablonneux. Cette espèce est nouvelle pour les Pyrénées où nous l'avons rencontrée jusqu'à $1600 \mathrm{~m}$ d'altitude.

Limnodrilus udekemainus Claparède, $1862 \mathrm{n}=\mathbf{2 4}$.

Répartition : cosmopolite.

France : Lorraine (Tétry, 1938) ; lac Léman (Juget, 1958 et 1967). 
Stations : 71, 72, 82, 83, 88 .

Nous avons rencontré $L$. udekemainus essentiellement en eau stagnante.

Limnodrilus claparedeianus Ratzel, $1868 \mathrm{n}=19$.

Répartition : cosmopolite.

France: Paris (Perrier, 1875) ; Lorraine (Tétry, 1938) ; lac Léman (Juget, 1958 et 1967) ; Dombes (Amoros et Juget, 1970) ; Seine, Yonne, Marne (Cohen, 1941).

Stations : $33,90,96$.

Psammoryctides barbatus (Grube, 1861 ) $\mathrm{n}=85$.

Répartition : Europe.

France : Paris (Perrier, 1875) ; Lorraine (Tétry, 1938) ; lac Léman (Juget, 1958 et 1967) ; lac d'Annecy (Juget, 1958) ; Yonne, Marne (Cohen, 1941).

Stations : $53,59,87$.

Nous avons rencontré cette espèce dans trois stations seulement ; elle formait là des populations très abondantes.

Potamothrix moldaviensis Vejdovsky et Mrazek, $1902 \mathrm{n}=6$.

Répartition : holarctique.

France : Saône (Juget, 1957).

Stations : 52, 74 .

Potamothrix hammoniensis (Michaelsen, 1901) $\mathrm{n}=61$.

Répartition : holarctique.

France : lac Léman (Juget, 1958 et 1967) ; lac d'Annecy (Juget, 1958 ).

Stations : $33,72$.

Nous avons prélevé cette espèce dans la zone profonde de deux lacs de barrage situés sur le Lot et la Garonne.

Ilyodrilus templetoni (Southern, 1909) $\mathrm{n}=12$. Sud.

Répartition : Europe, Asie, Amérique du Nord ?, Afrique du

France : lac Léman (Juget, 1957) ; Dombes (Amoros et Juget, $1970)$.

Stations : 82.

Peloscolex velutina (Grube, 1879) $\mathrm{n}=22$.

Répartition : Europe.

France : Lorraine (Tétry, 1938) ; Alsace (Moszinsky, 1938) ; lac Léman (Juget, 1958 et 1967) ; lac d'Annecy (Le Roux, 1907).

Stations : 31, 41, 51, 80, 81, 84, 93.

Cette espèce est localisée dans la partie supérieure des cours d'eau ; elle se rencontre également dans les fontaines où elle peut être très abondante (stations 93 où elle est le seul Oligochète 
présent). Bien qu'elle soit absente dans les torrents pyrénéens de haute montagne cette espèce se comporte comme une sténotherme d'eau froide.

Peloscolex ferox (Eisen 1879) $\mathrm{n}=34$.

Répartition : holarctique.

France : Lorraine (Tétry, 1938) ; lac Léman (Juget, 1958 et 1967); lac d'Annecy (Juget, 1958) ; Pyrénées (Juget et Giani, 1974).

Stations : $69,73,82,83$.

Ce Tubificidae est très peu représenté dans nos prélèvements alors que Juget et Giani (1974) l'ont récolté en abondance dans les lacs de haute altitude du Massif de Néouvielle.

\subsubsection{2. - Sous-famille des Aulodrilinae Brinkhurst, 1971.}

Aulodrilus pluriseta (Piguet, 1906) $\mathrm{n}=304$.

Répartition : cosmopolite.

France : lac Léman (Juget, 1958 et 1967) ; Pyrénées (Juget et Giani, 1974).

Stations : $38,52,54,63,66,67,68,69,70,71,72,73,74,75,76$, $77,78,82,83,90$.

A. pluriseta est le Tubificidae dominant de la partie moyenne des cours d'eau du Sud-Ouest de la France lorsque la pente et la vitesse du courant permettent l'envasement du lit; il colonise également tous les lacs de barrages mais contrairement à $T$. tubifex on ne le rencontre pas dans les mares.

Aulodrilus limnobius Bretscher, $1899 \mathrm{n}=33$.

Répartition : cosmopolite.

France : lac Léman (Juget, 1967).

Stations : 90.

2.2.3.3. - Sous-famille des Rhyacodrilinae Hrabe, 1963.

Rhyacodrilus coccineus (Vejdovsky, 1875) $\mathrm{n}=122$.

Répartition : Eurasie, Amérique du Nord, Australie.

France : ?.

Stations : 3, 7, 20, 23, 29, 30, 38, 43, 69, 83.

Cette espèce, nouvelle pour les Pyrénées, se rencontre jusqu'à $2000 \mathrm{~m}$ d'altitude dans certains torrents. Elle est le seul représentant de la famille des Tubificidae dans les sédiments grossiers des torrents pyrénéens. 
2.2.3.4. - Sous-famille des Branchiurinae Hrabe, 1966.

Branchiura sowerbyi Beddard, $1892 \mathrm{n}=125$.

Répartition : cosmopolite.

France : Alsace (Schweitzer, 1935) ; Lorraine (Tétry, 1938 ; Rémy, 1926) ; Canal du Midi à Toulouse (Despax, 1916) ; lac du Bourget (Léger, 1925) ; lac d’Annecy (Léger, 1924) ; lac Léman (Juget, 1967) ; Dombes (Amoros et Juget, 1970) ; Rhône (Perrier, 1909) ; Saône (Hesse et Paris, 1924 ; Juget 1957).

Stations : $57,70,71,72,73,74,75,76,77,78$.

Cette espèce est assez répandue en France et le Lot et l'Agoût sont deux stations nouvelles. Cette espèce vit en plaine dans les sédiments vaseux des rivières et des barrages; elle est vraisemblablement sténotherme d'eau chaude. Nous l'avons observée en phase de reproduction sexuée le 23-6-76 à la station 74 alors que l'eau était à la température de $31^{\circ}$ en surface et de $27^{\circ}$ au fond.

\subsection{4. - FAMILLE DES ENCHYTRAEIDAE}

Cette famille d'Oligochètes a très peu retenu notre attention ; toutefois à la liste fournie par Juget et Giani (1974) nous ajouterons une espèce.

Mesenchytraeus armatus Levinsen, 1884.

Répartition : Danemark, Allemagne, Pologne, Russie, Suisse, Irlande (Nielsen et Christensen, 1959).

France : ?.

Stations : 1, 41, 43, 44.

\subsection{5. - FAMILLE DES LUMBRICIDAE}

Eiseniella tetraedra (Savigny, 1826) $\mathrm{n}=321$.

Répartition : cosmopolite.

France : nombreuses fois signalée en France notamment par Cernosvitov (1931), Rémy (1932), Ginet (1962), Omodeo (1961), Beaucournu et Matile (1963), Juberthie et Mestrov (1965), Bou (1966), Juget (1967), Kiener et Ollier (1970), Gachet (1971), Tétry (1938).

Stations : 3, 4, 6, 7, 8, 9, 13, 16, 17, 19, 20, 21, 22, 24, 26, 27, $29,30,37,38,40,41,42,46,47,48,49,50,51,61,63,65,66,68$, $69,70,71,80,81,83,85,86,97$. 


\section{3. - RÊPARTITION DES OLIGOCHẼTES DANS LES PYRÊNÊES}

Le tableau I résume nos connaissances actuelles sur la répartition des Oligochètes dans les Pyrénées au-dessus de $1000 \mathrm{~m}$ d'altitude. Ce tableau récapitule les données fournies par le présent travail et il est complété par celles précédemment acquises (Juget et Giani 1974). La liste des Oligochètes aquatiques signalés dans les Pyrénées s'élève à 27 espèces - plus deux Enchytraeidae non déterminées des genres Achaeta et Marionina citées par Juget et Giani (1974). Il faut donc noter la faible diversité du peuplement d'Oligochètes dans les Pyrénées. Seules 22 espèces se rencontrent au-dessus de $1600 \mathrm{~m}$ d'altitude; cette diversité est encore plus restreinte dans les torrents puisque seulement 6 formes vivent dans les eaux courantes au-dessus de cette limite altitudinale. Parmi ces dernières 3 sont ubiquistes et se rencontrent également en eau stagnante jusqu'à $2300 \mathrm{~m}$ d'altitude (L. variegatus, $S$. heringianus, $M$. armatus).

Tableau I. - Répartition des Oligochètes dans les Pyrénées, au-dessus de $1000 \mathrm{~m}$ d'altitude. Les espèces marquées d'un astérisque sont nouvelles pour les Pyrénées.

\begin{tabular}{|c|c|c|c|c|}
\hline \multirow[t]{2}{*}{ Espèces } & \multirow{2}{*}{$\begin{array}{l}\text { Lacs et tourbières } \\
1000 \text { à } 2300 \mathrm{~m}\end{array}$} & \multicolumn{3}{|c|}{ Eaux courantes } \\
\hline & & 1000 & à $1600 \mathrm{~m}$ & $>1600 \mathrm{~m}$ \\
\hline Chaetogaster diaphanus & + & & & \\
\hline Chaetogaster diastrophus & + & & & \\
\hline Chaetogaster langi & + & & & \\
\hline Uncinais uncinata & + & & & \\
\hline Nais simplex & + & & & \\
\hline Tubifex ignotus & + & & & \\
\hline Peloscolex pyrenaicus & + & & & \\
\hline Peloscolex ferox & + & & & \\
\hline Aulodrilus pluriseta & + & & & \\
\hline Cernosvitoviella atrata & + & & & \\
\hline Cognettia glandulosa & $\dot{t}$ & & & \\
\hline Lumbriculus variegatus & + & & + & + \\
\hline Stylodrilus heringianus & + & & + & + \\
\hline Mesenchytraeus armatus* & + & & + & + \\
\hline Specaria josinae & + & & + & \\
\hline Nais communis & + & & + & \\
\hline Nais elinguis ${ }^{\star}$ & + & & + & \\
\hline Vejdovskyella comata & + & & + & \\
\hline Tubifex tubifex & + & & + & \\
\hline Haplotaxis gordioides* & & & + & \\
\hline Limnodrilus hoffmeisteri ${ }^{\star}$ & & & $\dot{t}$ & \\
\hline Nais bretscheri ${ }^{\star}$ & & & $\dot{t}$ & \\
\hline Nais pseudobtusa ${ }^{\star}$ & & & + & \\
\hline Slavina appendiculat $\alpha^{\star}$ & & & + & \\
\hline Rhyacodrilus coccineus * & & & $t$ & + \\
\hline Nais alpina* ${ }^{\star}$ & & & + & + \\
\hline Eiseniella tetraedra & & & + & $t$ \\
\hline
\end{tabular}


Le peuplement des lacs et des tourbières est plus diversifié puisqu'il comporte 19 espèces que nous avons rencontrées jusqu'à $2300 \mathrm{~m}$ d'altitude; parmi elles 8 vivent également en eau courante mais 5 ne dépassent pas alors $1600 \mathrm{~m}$ d'altitude; les trois autres sont les espèces ubiquistes déjà mentionnées. Cette plus grande diversité s'explique probablement par les températures supérieures atteintes par les eaux stagnantes. Cependant, les déversoirs des lacs, à altitude égale, ont des températures plus élevées que les torrents mais le peuplement en Oligochètes ne diffère pas. Il faut donc envisager également la nature du substrat comme facteur limitant. La vase des lacs, riche en matière organique, permet le peuplement en Tubificidae notamment. L'absence de sédiments fins et de substrat stable explique la faible représentation des Tubificidae dans les torrents. En fait, une étude quantitative montrerait probablement qu'au-dessus de $1600 \mathrm{~m}$ les Enchytraeidae deviennent le groupe dominant et constituent la plus grande partie du peuplement en Oligochètes des torrents.

\section{TRAVAUX CITÉS}

Aguesse (P.). 1957. - Complément à l'inventaire de la faune invertébrée des eaux camarguaises. La Terre et la Vie, 104:241-252.

Amanieu (M.). 1969. - Recherches écologiques sur les faunes des plages abritées de la région d'Arcachon. Helgolander wiss Meeresunters, $19: 455-557$.

Amoros (C.) et Juget (J.). 1970. - Données préliminaires sur la faune planctonique et benthique de deux étangs piscicoles de la Dombes (Ain). Annls limnol., 6 (2) : 215-227.

Beaucournu (L.) et Matrle (L.). 1963. - Contribution à l'inventaire faunistique des cavités souterraines de l'Ouest de la France. Ann. Spéléol., $18: 343-357$ et 519-531.

BeRTHÉlEMy (C.). 1966. - Recherches écologiques et biogéographiques sur Ies Plécoptères et Coléoptères d'eau courante (Hydraena et EIminthidae) des Pyrénées. Annls limnol., 2 (2) : 227-458.

Berthélemy (C.) et Laur (C.) . 1975. - Plécoptères et Coléoptères aquatiques du Lot (Massif Central Français). Annls limnol. ,11 (3) : 263-285.

Bou (C.). 1966. - Faune souterraine du Sud-Ouest du Massif Central. I. Contribution à la connaissance des Invertébrés cavernicoles. Ann. Spéléol., 21 (3) : 689-706.

BrinkhuRST (R. O.). 1966. - Oligochaeta. In : Limnofauna Europaea, J. IIllies éd., A. Fisher Verlag, Stuttgart : 110-117.

Brinkhurst (R. O.), Jamieson (B. G. M.). 1971. - Aquatic Oligochaeta of the world. Oliver and Boyd., Edinburgh, $860 \mathrm{p}$.

Brunotre (C.). 1892. - Deux stations nouvelles du Pheoryctes menkeanus (Hoffmeister). Rev. Biol. du Nord de la France, V : 120122. 
Cernosvitov (L.). 1931. - - Notes sur les Oligochètes des grottes de Sainte-Reine près de Toul. Arch. Zool. Exp. Gén. Notes et Revues, $71: 62-66$.

Cohen (F.). 1941. - Oligochètes, Hirudinées, Turbellariés de la Seine et de quelques-uns de ses affluents de la région parisienne. Commission du Bassin de la Seine. Travaux de la rubrique Biologie (Etudes biocénotiques). Cahier $\mathrm{n}^{\circ} 2$. Chez Gauthier-Villars, Paris.

Décamps (H.). 1967. - Écologie des Trichoptères de la vallée d'Aure (Hautes-Pyrénées). Annls limnol., 3 (3) : 399-577.

DelPHy (J.). 1921. - Etudes sur l'organisation et le développement des Lombriciens Limicoles Thallassophiles. Travaux du laboratoire de Zoologie comparative de l'école pratique des Hautes Études, $136 \mathrm{p}$.

Despax (M.). 1916. - Une nouvelle station française de Branchiura sowerbyi Beddard. Bull. Soc. Zool. de France, XLI : 46-48.

Despax (M.). 1927. - Recherches sur les facteurs de la distribution des organismes. Bull. Biol., 2 : 126-142.

Ferronnière (G.). 1899. - Contribution à l'étude de la faune de la Loire-Inférieure (Annélides, Oligochètes). Bull. Soc. Sci. Ouest Fr., 9 : 227.

Florentin (R.). 1899. - Étude sur la faune des mares salées de Lorraine. Ann. Sci. Nat., 8 (X) : 209-349.

Gachet (M.). 1971. - Etude de la fluctuation de la faune ripicole macroscopique d'un étang astatique de la région grenobloise. Trav. Lab. Hydrobiol., 62 : 17-57.

Giard (A.). 1882. - Sur le Crenothrix kubniana cause de l'infection des eaux de Lille. $C . R$. de l'Ac. Sci., 31 juillet 1882.

Giard (A.). 1889. - Sur l'habitat de Phreoryctes menkeanus Hoff. Bull. Sci. France Belgique, XX.

Ginet (R.). 1962. - Faune cavernicole du Jura Méridional et des chaînes subalpines dauphinoises. II. Contribution à la connaissance des Invertébrés. Ann. Spéléol., 16 (3) : 303-325.

Hartmann (G. I.). 1923. - Sur l'habitat de Haplotaxis gordioides. Bull. Soc. Zool. Fr., XLVIII : 138-140.

Hesse (E.) et PAris (P.). 1924. - Sur la présence de Branchiura sowerbyi (Bedd.) dans le département de la Côte-d'Or. Annales de l'Université de Grenoble, XXXIV : 1-5.

Hubault (E.). 1927. Contribution à l'étude des Invertébrés torrenticoles. Bull. Biol. France Belgique, suppl. 9 : 1-389.

Juberthie (C.) et Mestrov (M.). 1965. - Sur les Oligochètes terrestres des sédiments argileux des grottes. Ann. Spéléol., 20 (2) : 209236.

JugEt (J.). 1957. - Quelques aspects de la faune limicole des environs de Saint-Jean-de-Losne. Trav. Lab. Zool. Stn. Aquic. Grimaldi, Dijon, 22 : 1-3.

Juget (J.). 1958. - Recherche sur la faune de fond du Léman et du lac d'Annecy. Ann. St. Cent. Hydrobiol. appl., $7: 9-56$.

Juget (J.). 1959. - Recherches sur la faune aquatique de deux grottes du Jura. Ann. spéléol., $14: 391-401$.

JugET (J.). 1967. — Quelques données nouvelles sur les Oligochètes du Léman : composition et origine du peuplement. Annls limnol., $3: 217-229$. 
JugEt (J.) et GiANi (N.). 1974. - Répartition des Oligochètes lacustres du Massif de Néouvielle (Hautes-Pyrénées) avec la description de Peloscolex pyrenaicus, n. sp. Annls limnol., 10 (1) : 33-53.

Kiener (A.) et Oldier (J.). 1970. - Contribution à l'étude écologique et biologique de la rivière le Gapeau (Var). Hydrobiologia, 36 : 189-251.

LAFON (J.). 1953. - Recherches sur Ia faune aquatique littorale du Rhône à Lyon. Bull. mens. Soc. Linn. Lyon, 22 (2) : 36-46.

Lasserre (P.). 1966. - Oligochètes marins des Côtes de France. I. Bassin d'Arcachon : systématique. Cah. Biol. Mar., VII : 295-317.

Lasserre (P.). 1967. - Oligochètes marins des Côtes de France. II. Roscoff, Penpoull, Etangs saumâtres de Concarneau : systématique, écologie. Cah. Biol. Mar., VIII : 273-293.

Lavandier (P.). 1974. - Écologie d'un torrent pyrénéen de haute montagne. I. Caractéristiques physiques. Annls limnol., 10 (2) : 179219.

LÉGER (L.). 1924. -- La répartition de Branchiura sowerbyi et son rôle en économie piscicole. C. R. Ac. Sci., $178: 240$.

LÉGER (L.). 1925. - Sur deux stations nouvelles de Branchiures en Savoie et en Dauphiné. Trav. Lab. Piscicult. Grenoble, 15/16 : 29-32.

LE Roux. 1907. - Recherches biologiques sur le lac d'Annecy. Ann. Biol. Lacustre, Bruxelles, 2 : 220-387.

Monard (A.). 1928. - Note sur la faune de quelques lacs des Pyrénées. Bull. Soc. Zool. France, 53 : 243-261.

Moniez (R.). 1888. - Faune des eaux souterraines du département du Nord et en particulier de la ville de Lille. Revue Biologique $d u$ Nord de la France, 4 : 142-153.

Moszinsky (A.). 1938. - Oligochètes de biotopes hypogés de la vallée du Rhin, d'Alsace et des régions limitrophes. Arch. Zool. Exp. Gén., $8: 1-10$.

Nielsen (C. O.) et Christensen (B.). 1959. - The Enchytraeidae, critical revision and taxonomy of European species. Nat. Jutl., 8/9:1-160.

OMoneo (P.). 1961. - Oligocheti della Francia meridionale e di localite limitrofe. Mém. Mus. Civ. Storia Nat. Verona, 9 : 67-95.

Perrier (E.). 1875. - Sur le Tubifex umbellifer. Arch. Zool. Exp. Gén. Noles et Revues. T. IIV.

Perrier (L.). 1909. - Une station rhodanienne de Branchiura sowerbyi (Bedd.) Annales de l'Université de Grenoble, XXI : 235-242.

Pesson (P.). 1935. - Notes de faunistique armoricaine (2 note). Bull. Soc. Sci. Bretagne, 12 : $190-197$.

Pesson (P.). 1938. - Notes de faunistique armoricaine $\left(4^{\mathrm{e}}\right.$ note). Bull. Soc. Sci. Brelagne, 15 : 39-48.

PUJol (J.-Y.). 1975. - Quclques aspects de la biologie des Drusinae (Trichoptères) et influences humaines sur un cours d'eau de montagne. Thèse 3" cycle, Toulouse, $133 \mathrm{p}$.

RÉmy (P.). 1926. - Note sur la distribution géographique de Branchiura sowerbyi Beddard. Ann. Biol. Lacustre, Bruxelles, 15 : 55-60.

Rémy (P.). 1932. - Trois espèces intéressantes de la faune vosgienne. Bull. Soc. Hist. Nat. Moselle, 4 (9) : 47-54.

RÉmy (P.). 1943. - Notes faunistiques. I. Vers et Mollusques. Bull. Soc. Linn. de Lyon, 12 (9) : 139-142.

Richard (J.). 1897. - Sur un Oligochète et quelques Entomostracés rares des environs de Paris. Bull. Soc. Zool. France, XXII : 224227. 
Schodduyn (R.). 1925. - Contribution à l'étude biologique du Canal de Roubaix (Nord de la France). Ann. Biol. Lacustre, Bruxelles, $14: 89-110$.

Schodduy (R.). 1926. - Matériaux pour servir à l'étude biologique des cours d'eau de la Flandre Française. Ann. Biol. Lacustre, Bruxelles, 14 : 281-350.

Schodduyn (R.). 1926. - Contribution à l'étude du plancton du lac de Lourdes (Hautes-Pyrénées). Ann. Biol. Lacustre, XIII (1) : 145204.

Schweitzer (A.). 1935-36. - Quelques Oligochètes d'Alsace. Bull. de l'Assoc. Philomatique d'Alsace et de Lorraine. T. VIII, fasc. 3 : 219.

TÉTry (A.). 1938. - Contribution à l'étude de la faune de l'Est de la France (Lorraine). Thèse, Nancy, 1-453 p.

Thomas (A. G. B.). 1976. - Diptères torrenticoles peu connus. IV. Les Athericidae (écologie et biologie) du Sud de la France (Brachycera, Orthorrapha). Annls limnol., 12 (2) : 175-211.

Vandel (A.). 1922. - Sur la faune d'eau douce des Pyrénées-Orientales. Bull. Soc. Zool. Fr., 47 : 163-170. 